\title{
DESCRIPTION D'UNE NOUVELLE ESPECE DE TRYPANOSOME
}

TRYPANOSOMA CROCIDURAE

\section{CHEZ UNE MUSARAIGNE (CROCIDURA RUSSULUS)}

\author{
Par E. BRUMPT
}

En examinant, en juillet 1920, le sang de trois musaraignes (Crocidura russulus Hermann, 1780), capturées à Arcueil, à quelques kilomètres de Paris, et mises à ma disposition par mon collègue, le professeur agrégé Ch. Joyeux, j'ai rencontré deux fois un trypanosome du type Lewisi dont je vais donner une description sommaire.

A l'état frais le trypanosome ressemble beaucoup par son agilité à celui du rat. Après coloration par la méthode panoptique de Pappenheim, ce parasite prend les aspects représentés sur la figure 1 .

Il mesure, flagelle compris, de 31 à $35 \mu$ de long sur 2 à $2,5 \mu$ de large environ au niveau de son plus grand diamètre situé à la hauteur du noyau.

Le corps se termine généralement par une pointe fine, droite ou flexueuse. Le tableau ci-dessous, nous indique les mensurations prises sur dix exemplaires et évaluées en microns.

DIMENSIONS DU TRYPANOSOMA CROCIDURA

\begin{tabular}{|c|c|c|c|c|c|c|c|c|c|}
\hline 造 & 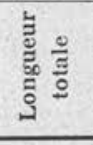 & 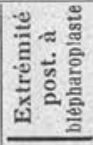 & 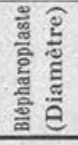 & 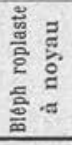 & 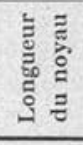 & 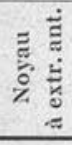 & 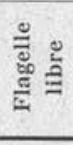 & 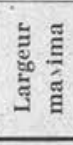 & 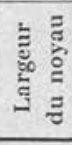 \\
\hline I. & 31 & 5 & 0,50 & 6 & 3 & 12 & 5,5 & 2,25 & 1,75 \\
\hline II. & 34 & 7 & 0,75 & 6 & 4,25 & 10 & 6 & 2 & 1,50 \\
\hline III. & 34,75 & 7,5 & 0,75 & 7 & 3 & 9 & 7,5 & 3 & 1,50 \\
\hline IV. & 34 & 7,5 & 0,75 & 5,5 & 3,75 & 8 & 8,5 & 2 & 1,50 \\
\hline V. & 34,25 & 7,5 & 0,75 & 5,5 & 3 & 10 & 7,5 & 2,5 & 1,50 \\
\hline VI. & 35 & 7 & 0,75 & 7 & 3,75 & 10 & 6,5 & 2 & 1,25 \\
\hline VII. & 34,25 & 8 & 0,75 & 5 & 3 & 9,5 & 8 & 2 & 1,50 \\
\hline VIII. & 34 & 11 & 0,75 & ? & $?$ & $?$ & 5 & 2,5 & ? \\
\hline IX. & 34,75 & 8,5 & 0,75 & 5,5 & 3 & 10 & 7,5 & 2 & 1,50 \\
\hline X. & 33,25 & 7 & 0,75 & 6 & 3,5 & 7 & 9 & 2 & 1,25 \\
\hline
\end{tabular}

Le protoplasma se colore en rose violacé faible ; il renferme de très fines granulations.

Le noyau, long de $3 \mu$ à $4,25 \mu$, est parfois peu visible; il est situé un peu au-dessus du milieu du corps, mais il est beaucoup

Annales de Parasitologie, T. I, No $3 .-$ Août 1923. 
moins rapproché dé l'extrémité flagellaire que chez le Trypanosoma Lewisi (fig. 2). Il est constitué par des granulations chromatiques d'un rouge violacé, généralement plus foncé que le cytoplasme.

Le blépharoplaste, sphérique ou ovalaire, mesure de $1 / 2$ à $3 / 4$ de $\mu$; il est situé à une distance de 5 à $11 \mu$ de l'extrémité postérieure ; il est généralement entouré d'une zone claire au niveau de laquelle le flagelle s'arrête. Le flagelle libre mesure de 5 à $9 \mu$. La membrane ondulante est peu plissée comme chez tous les trypanosomes du type Lewisi.

Ces parasites semblent avoir un protoplasme assez consistant, car

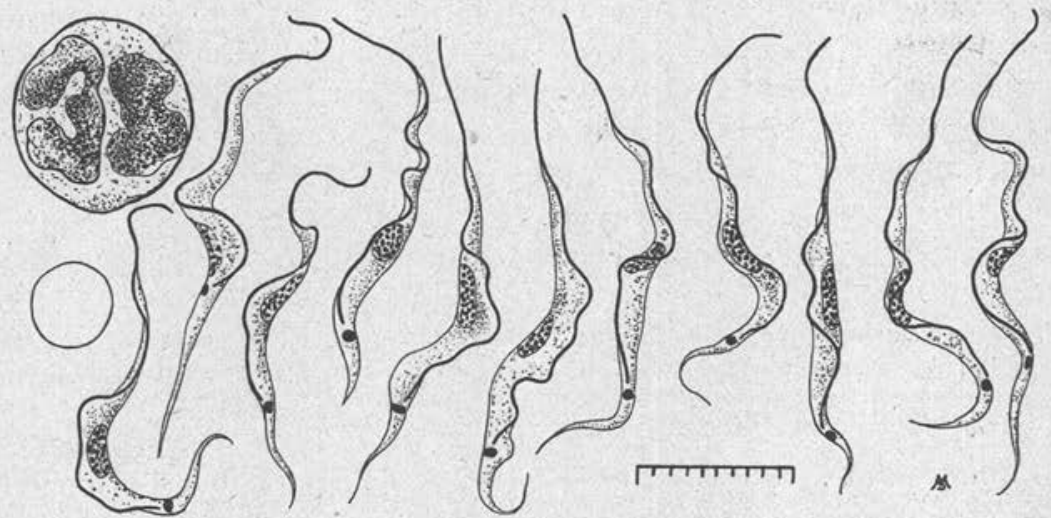

Fig. 1. - Trypanosoma crociduræe n. sp. Sang périphérique de la musaraigne 741, $\mathrm{V}$; remarquer la gracilité du parasite et la position sub-centrale du noyau.

ils subissent l'étalement sur les frottis sans altération notable, contrairement à ce qui se passe pour certains trypanosomes comme le $T$. Cruzi et divers trypanosomes de batraciens, par exemple.

Je n'ai observé aucune forme de multiplication, ce qui indique qu'il s'agissait d'infections anciennes.

Le trypanosome de la musaraigne ne s'est pas montré infectieux pour un jeune rat âgé de six semaines, ni pour les jeunes souris âgées de 2 jours à six semaines ( 8 expériences négatives), même par la voie péritonéale. Il coexistait dans le sang de ces insectivores avec une Grahamella, que je n'ai pas réussi à transmettre aux rats ni aux souris.

Le Trypanosoma crociduræ était peu abondant dans le sang $d t$ deux animaux infectés. L'un d'eux $(742$, V.) présentait un parasite par deux champs microscopiques (400 diam.), l'autre $(764$, V.) a montré quelques rares formes grêles dans le sang du cœur.

L'identification de ce parasite est difficile, comme celle de tous les trypanosomes du type Lewisi; il semble cependant assez différent 
de ce dernier représenté sur la figure 2, dont les frottis ont été confectionnés le même jour que ceux de la musaraigne et colorés avec les mêmes réactifs. Il est également différent du Trypanosoma soricis décrit par Watson et Hadwen et trouvé par ees auteurs dans le sang d'une musaraigne du Canada: Sorex vagrans Baird, 1857. Deux musaraignes sur cinq étaient infectées. Le trypanosome décrit par les auteurs canadiens, est, en effet, plus petit et plus gros que celui dont je viens de donner la description; il présente une longueur totale de $17,5 \mu$, possède un flagelle libre court et un noyau central. D'autre part, à en juger par les deux bonnes microphoto-

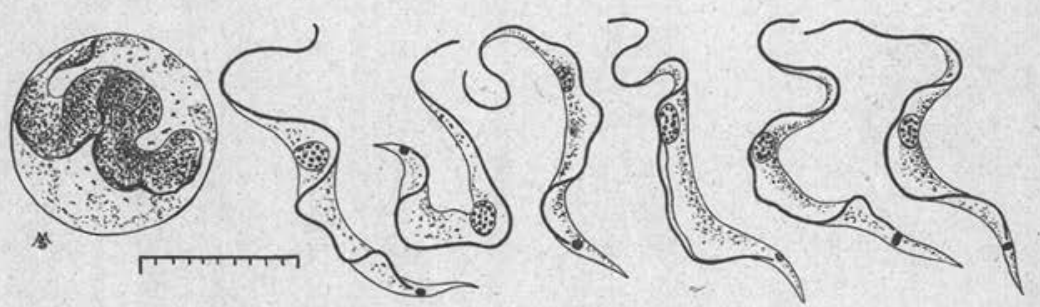

Fig. 2. - Trypanosoma Lewisi. Sang périphérique d'un jeune surmulot de trois mois environ présentant une infection naturelle ; remarquer la position du noyau généralement très rapproché de l'extrémité flagellaire.

graphies jointes au travail de ces auteurs, leur parasite est beaucoup plus trapu que le $T$. crociduræ.

Faute de musaraignes neuves et de milieux appropriés, il m'a été impossible d'étudier les formes de multiplication et les formes de culture du Trypanosoma crociduræ.

La transmission de ce parasite, comme celle de tous les trypanosomes non pathogènes du type Lewisi trouvés chez les rongeurs et les taupes, doit s'effectuer par l'intermédiaire des puces. La troisième musaraigne examinée par moi, celle dont le sang ne montrait pas de trypanosome, hébergeait quelques puces aveugles pourvues d'un peigne à la partie postérieure de la tête. Les préparations de ces puces n'ayant pu être retrouvées, il m'est impossible d'en donner la détermination exacte et leur petit nombre m'a empêché de les disséquer pour savoir si elles hébergeaient des formes évolutives du T. crociduræ.

\section{Bibliographie}

Laveran (A.) et Mesnil (F.). - Trypanosomes et Trypanosomiases, 2e édit., Masson et Cie éditeurs, Paris, 1912.

WAtson (E.) et Hadwen (S.). - Trypanosomes found in Canadian mammals. Parasitology, V, 1912-1913, p. 25, pl. II, fig. 8 et 9.

Laboratoire de Parasitologie de la Faculté de médecine de Paris. 